Annales Geophysicae (2002) 20: 1783-1793 (c) European Geosciences Union 2002

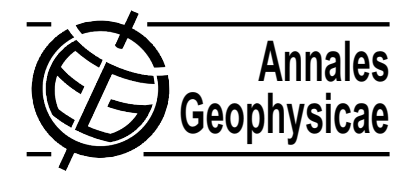

\title{
Influence of magnetospheric processes on winter HF radar spectra characteristics
}

\author{
R. André ${ }^{1}$, M. Pinnock ${ }^{2}$, J.-P. Villain ${ }^{1}$, and C. Hanuise ${ }^{3}$ \\ ${ }^{1}$ LPCE/CNRS, 3 A Av. de la Recherche Scientifique, 45071 Orléans Cedex, France \\ ${ }^{2}$ British Antarctic Survey, High Cross, Madingley Road, CB3 OET Cambridge, UK \\ ${ }^{3}$ LSEET/CNRS, Université de Toulon et du Var, BP 132, 83957 La Garde Cedex, France
}

Received: 13 September 2001 - Revised: 4 March 2002 - Accepted: 13 March 2002

\begin{abstract}
This study investigates further the relationship between regions of the magnetosphere and the characteristics of HF radar Doppler spectra recorded in the ionospheric projection of those regions. It builds on earlier work, which has reported a relationship between the Doppler spectral width and the ionospheric projection of the magnetospheric cusp region, by introducing novel techniques for classifying the Doppler spectra recorded by the SuperDARN radars. We first review the geophysical factors that can condition the characteristics of the autocorrelation function (ACF) data produced by the radars. This leads to a classification scheme of the ACF data which is then applied to a large database compiled from winter data taken by the Northern Hemisphere SuperDARN radars. This statistical study shows that the ACF characteristics are not randomly distributed in space, but rather are spatially organized in the ionosphere. This paper suggests that these regions are ordered primarily by the low energy $(\approx 1 \mathrm{keV})$ electron precipitation region and the presence of intense ULF wave activity.
\end{abstract}

Key words. Ionosphere (auroral ionosphere; ionospheremagnetosphere interactions; plasma convection)

\section{Introduction}

The SuperDARN HF radar network (e.g. Greenwald et al., 1995) has proved to be a valuable tool for investigating features of ionospheric plasma convection at high latitudes. It is known that these features relate to conditions in the solar wind (e.g. Heppner, 1972) or to the close coupling of the magnetosphere/ionosphere system (e.g. convection features associated with the substorm current wedge; Opgenoorth et al., 1983). Many of the results published from the SuperDARN radars use only their prime data product, the determination of the line-of-sight Doppler velocity, from which the bulk motion of the ionosphere is inferred. However, the radars also routinely provide data, giving a more detailed de-

Correspondence to: C. Hanuise (hanuise@ cnrs-orleans.fr) scription of the spectral characteristics of the backscattered signal. This paper introduces new techniques for utilizing this information and reports on the initial findings from a database of spectral characteristics and the relationship to magnetospheric boundaries projected into the ionosphere.

Earlier SuperDARN radar studies have discovered relationships between the spectral characteristics of backscatter and specific magnetospheric regions, in particular, the Doppler spectral width (the full width at half power of a single component spectra; see Baker et al., 1995). Baker et al. (1990, 1995) and Rodger et al. (1995) have shown that the radar spectral width is greatly enhanced in the ionospheric footprint of the cusp. Baker et al. (1995) also showed that the radar spectra were characterized by multi-component spectra. Several studies have used the Doppler spectral width parameter to identify the cusp, and to derive more information about solar wind-magnetosphere-ionospheric coupling, such as the temporal evolution of the reconnection rate at the magnetopause (Baker et al., 1997; Pinnock et al., 1999). On the nightside, some studies have related the increase of spectral width as a function of latitude with the central plasma sheet (CPS)/boundary plasma sheet (BPS) separatrix (Dudeney et al., 1998).

Recently, André et al. (1999, 2000a) have provided a physical explanation for the enhanced Doppler spectral width observed in the cusp and André et al. (2000b) have extended this work to review several factors that condition the radar spectra. These factors may be divided into microscopic ones associated with the plasma physics of irregularities and macroscopic factors which are themselves divided into spatial and temporal variations of the electric field within the radar's range gate. When it is known that the macroscopic factors are negligible, then the microscopic factors can be studied by detailed analysis of the ACF, and factors such as the plasma diffusion coefficient derived (e.g. Villain et al., 1996).

André et al. (2000b) showed that spatial variations within the radar range gate (cell size typically $45 \times 100 \mathrm{~km}$ ) arise from both the large-scale convection pattern, which intro- 
duces velocity gradients within a range gate, and smaller scale features, such as vortices associated with field-aligned currents (Schiffler et al., 1997; Huber and Sofko, 2000). André et al. (2000a) also examined the impact of temporally varying electric fields, where the period of the variations was less than the integration time of the radar. In particular, they demonstrated that intense wave activity in the Pc1 frequency band can explain all the ACF characteristics observed in the cusp. The effect of under-sampling the plasma velocity variations is to introduce several unreal components in the Doppler spectrum, which lead the routine analysis to compute a very high spectral width (André et al., 1999, 2000a).

As detailed in André et al. (2000b), each geophysical feature introduces specific signatures in the ACF, such as a modulation of its temporal evolution of phase and power, which can be determined by the routine analysis. In this paper, we introduce an ACF classification scheme that separates the multi-component from the single component spectra. This technique is then applied to a large database. The derivation of the database and its characteristics are described in Sect. 2 and the ACF classification scheme is introduced in Sect. 3. Section 4 discusses the well-defined distribution of these ACF classes over the auroral zone, and their relationship to the large-scale convection pattern and low energy electron precipitation.

\section{Statistical study}

\subsection{Data selection}

SuperDARN is an HF radar network that was constructed primarily to measure the large-scale convection pattern in a plane perpendicular to the magnetic field (Greenwald et al., 1995) over the ionospheric auroral zone and polar cap. Each radar has an array of $16 \log$-periodic antennas, from which 16 beams (each $3.3^{\circ}$ wide) are formed through electronic phase delay switching. From the transmission of a multiple pulse scheme, repeated through the integration time, the radars measure the complex autocorrelation function (ACF) of the signal backscattered at each range gate (usually from $180 \mathrm{~km}$ to $3330 \mathrm{~km}$ in 70 range gates) by field-aligned irregularities occurring in the radar beam. For each range gate, this ACF is analysed routinely by a basic method (FITACF) (Villain et al., 1987; Baker et al., 1995) which extracts the power, the line-of-sight Doppler velocity of the irregularities and the spectral width. In their common sounding mode, the radars have an integration time on each beam of $7 \mathrm{~s}$, and it takes $120 \mathrm{~s}$ to scan all 16 beams. The radars are designed to run $24 \mathrm{~h}$ a day and 365 days a year.

A database has been compiled from data recorded by 6 radars located in the Northern Hemisphere (in Finland (Hankasalmi), Iceland (Thykkvibaer and Stokkseyri) and Canada (Goose Bay, Kapuskasing and Saskatoon). To avoid any seasonal variations, we have chosen data only from winter (from November 1995 to April 1996 and from October 1996 to March 1997). Winter coincides with the seasonal maximum in the data rate from these radars (Ruohoniemi and Greenwald, 1997).

We have selected data only from the F-region to ensure that the irregularities are moving with the bulk plasma flow (Villain et al., 1985). This selection is satisfied most of the time by taking only data located at more than $900 \mathrm{~km}$ from the radar. One has to note that this criteria may not reject all E-region data observed at a large distance ( $1 \frac{1}{2}$ hop propagation mode). Only ionospheric data with a signal to noise ratio greater than $3 \mathrm{~dB}$ are used. We have rejected scatter that could come from the ground, identified as having both a low velocity $(<50 \mathrm{~m} / \mathrm{s})$ and spectral width $(<50 \mathrm{~m} / \mathrm{s})$. To avoid any contamination from data recorded during specific radar operating modes (for example, with a shorter integration time), we have taken only periods when radars were running in the common mode (integration time of 6 or $7 \mathrm{~s}$ ). All these data are mapped onto a grid defined in geomagnetic coordinates which has a resolution of $1^{\circ}$ in Magnetic Latitude (MLAT) and $30 \mathrm{~min}$ in Magnetic Local Time (MLT), using the AACGM coordinate system which is based on the earlier PACE coordinate system of Baker and Wing (1989). For the twelve months of radar operation, the database holds about $22.310^{6}$ ACFs.

The data has been sorted by the prevailing IMF conditions as measured by the Wind satellite for periods when it is in the solar wind. Wind data are averaged over $30 \mathrm{~min}$ and shifted in time according to the distance from the satellite to the Earth in the GSM $Y$ plane and the solar wind velocity. The data can also be sorted by the planetary magnetic activity index $\left(K_{p}\right)$. The average IMF is found to be very small $\left(B_{x} \approx 0.7 \mathrm{nT}, B_{y} \approx-0.7 \mathrm{nT}\right.$ and $B_{z} \approx 0.3 \mathrm{nT}$ ), and $K_{p}$ is 2+. Both IMF components and $K_{p}$ are normally distributed around their mean values, as shown in Fig. 1.

The distribution of data in a geomagnetic latitude MLT frame is very similar to that obtained by Ruohoniemi and Greenwald (1997). On the dayside, the number of points is highest in a region that includes the cusp, the low-latitude boundary layer (LLBL) and the mantle. It is well-known that the equatorward boundary of the cusp is a well-defined target for HF radars (Milan et al., 1999). On the nightside, the maximum in the scatter rate is co-located with the poleward half of the auroral oval. In the dawn sector there is a deep minimum in the scatter rate that can be explained by an enhancement in HF wave absorption due to intense electron precipitation. In the dusk sector there is an increase in the scatter rate that could be related to scintillations from subauroral irregularities (Basu, 1978; Fremouw and Lansinger, 1981), as shown by Ruohoniemi et al. (1988).

\subsection{Spectral width}

In each point of the defined grid, one can construct a distribution function for each parameter extracted from the ACF, and compute its mean value. In order to avoid any bias given by the tails of the distribution, we have discarded points in the distribution that contain less than a defined threshold. The threshold used in this paper is 500 points, which represents 

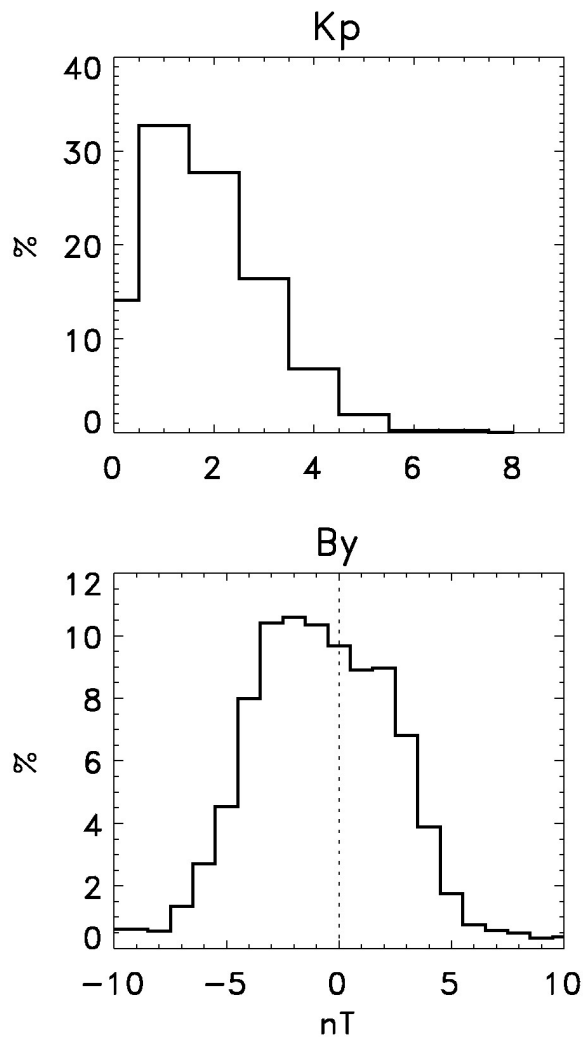

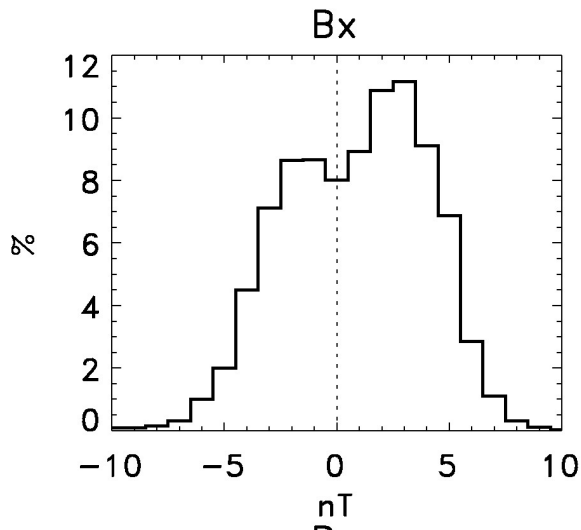

$\mathrm{Bz}$

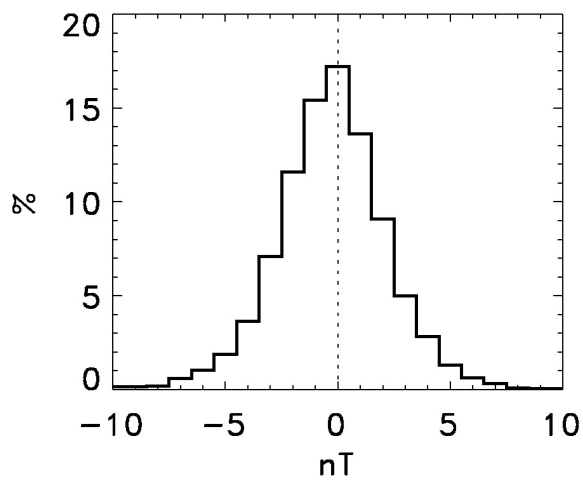

Fig. 1. Distributions of values for $K_{p}$ and the three IMF components corresponding to the whole period included in the database. They are normal distributions centred around very small average values $\left(B_{x} \approx 0.7 \mathrm{nT}, B_{y} \approx 0.7 \mathrm{nT}\right.$ and $B_{z} \approx 0.3 \mathrm{nT}$ ), and $K_{p}$ is $2+$. about $2 \%$ of the data in one distribution function (taking into account the total number of points and that the grid is defined by 48 MLT $\times 20$ MLAT useful points). Reducing this threshold gives more unrealistic values in the computed mean value, and increasing this threshold decreases the number of points in the geomagnetic grid where this mean value can be computed. The robustness of our results has been checked by plotting for varying threshold levels and it has been found that they are not overly sensitive to the threshold value.

Figure 2a displays the mean value of the spectral width. The black line represents the average position of the poleward boundary of the auroral oval, as modelled by Holzworth and Meng (1975) for moderate magnetic activity. The average spectral width is very high $(>350 \mathrm{~m} / \mathrm{s})$ in a region that corresponds to the cusp, as expected (Baker et al., 1990, 1995). This quantity has a high value (about $250 \mathrm{~m} / \mathrm{s}$ ) along a region shaped like the auroral oval and located poleward of its modelled poleward boundary. This statistical observation is in very good agreement with the case study in the nightside sector published by Lester et al. (2001). Dudeney et al. (1998) have also shown in a case study that the boundary between high and low spectral widths on the nightside may be collocated with the boundary between the Central Plasma Sheet (CPS) and the Boundary Plasma Sheet (BPS). Finally, the spectral width is low (around $150 \mathrm{~m} / \mathrm{s}$ ) equatorward of the low-latitude boundary of the Feldstein auroral oval and in the high-latitude polar cap.

\section{ACF classification}

\subsection{Basics}

As discussed in the Introduction, several factors can increase the Doppler spectral width. Some of these factors lead to a multi-component Doppler spectra (e.g. intense Pc1 wave activity; vortices with scale sizes comparable to the radar sampling cell), whereas others lead to one wide component (certain areas of the large-scale convection pattern; microscopic plasma turbulence arising from the plasma physics of the irregularities). In the case of a multi-component spectrum, the ACF phase and power arising from the component with the greatest power are modulated by the subsidiary components (Barthes et al., 1998). This modulation artificially increases the decorrelation time of the ACF power. Since the standard analysis fits this function with a mono-exponential or a Gaussian function, this spectrum leads to the determination of a high spectral width with a large error. Since the ACF phase is also modulated, the linear fit that extracts the Doppler velocity has also a relatively large error. Therefore, one can evaluate if the Doppler spectrum is composed of oneor several peaks by checking the errors performed on these fits by the standard analysis routine. Figures $2 b$ and $c$ show the average values of the standard deviation associated with the fit performed on the ACF phase (Fig. 2b) and power (Fig. 2c). These errors are lower at both low and very high latitudes and much higher in the dayside, while around the cusp region the standard deviation in the power fit shows a maximum. This 

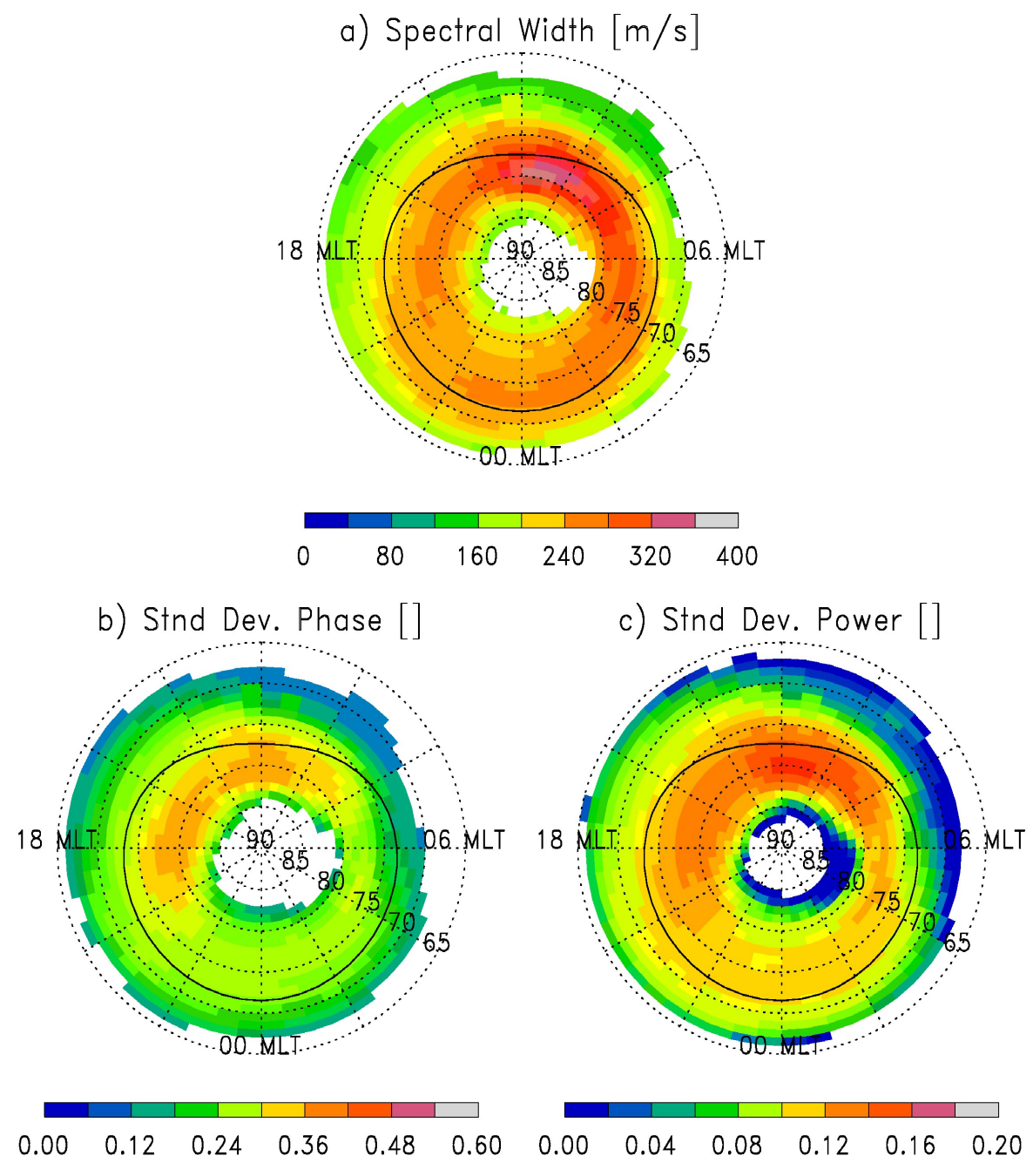

Fig. 2. Mean value of the (a) ACF spectral width, (b) errors on the fit performed on the ACF phase and (c) errors on the fit performed on the ACF power. The black line represents the average position of the poleward boundary of the auroral oval, as modelled by Holzworth and Meng (1975) for moderate magnetic activity.

last feature is consistent with the multi-component spectra induced by low-frequency wave activity (André et al., 1999, 2000a).

These patterns clearly demonstrate that these errors are not randomly distributed. This strongly suggests that they reveal an area where the recorded Doppler spectra are likely to have several components. We have checked to ensure that the regions of high standard deviation are not associated with an individual radar's contribution to the database.

\subsection{Method}

One can classify the characteristics of each ACF using the data derived from the standard analysis (spectral width and standard deviations), in order to separate ACFs that have multiple velocity components from those that have a single component.

We have defined three basic classes of ACF, summarised in Table 1. The class "S" ACF has a low spectral width and also low standard deviations on the phase and power fits. The associated spectrum should have one narrow component. At the opposite, the class " $m$ " ACF has high standard devia-
Table 1. Characteristics of the three classes of ACF according to the spectral width and standard deviation on phase and power

\begin{tabular}{ccc}
\hline Class & Spectrum & Conditions \\
\hline \multirow{3}{*}{$\mathrm{S}$} & & Narrow width, single \\
& Stnd. Dev. Phase $<0.30$ \\
& Stnd. Dev. Pow. $<0.15$ \\
& & Width $<200 \mathrm{~m} / \mathrm{s}$ \\
$\mathrm{T}$ & Large width, single & Stnd. Dev. Phase $<0.30$ \\
& Stnd. Dev. Pow. $<0.15$ \\
& component spectrum & Width $>200 \mathrm{~m} / \mathrm{s}$ \\
$\mathrm{m}$ & & Stnd. Dev. Phase $>0.30$ \\
& Multi-component & Stnd. Dev. Pow. $>0.15$ \\
& spectrum & Width $>200 \mathrm{~m} / \mathrm{s}$ \\
\hline
\end{tabular}

tions on both phase and power fits and also a high spectral width. The associated spectrum is likely to have several components. Finally, the class "T" ACF is characterized by a high spectral width but low standard deviations. The associated spectrum would have a single wide component. ACFs 

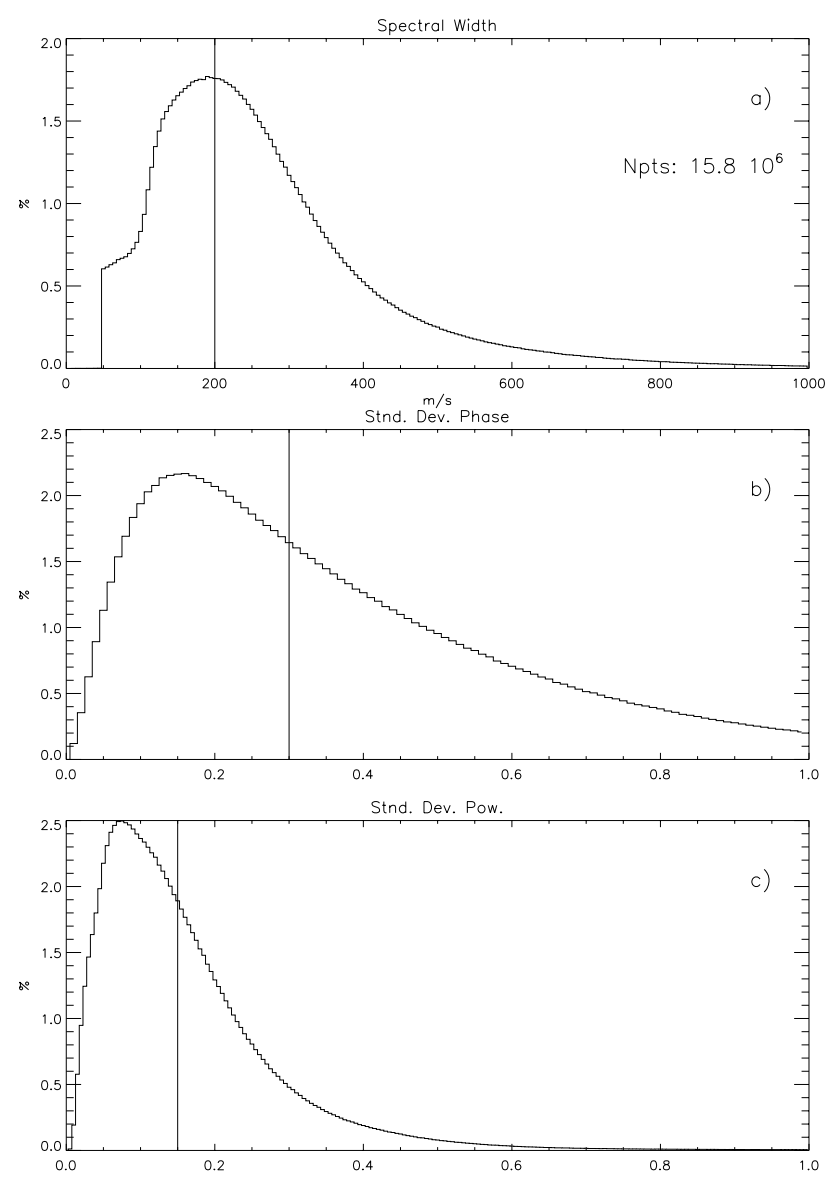

Fig. 3. Density probability of (a) spectral width, (b) standard deviation on the phase and (c) standard deviation on the power. Vertical lines show the thresholds used in this classification scheme for each parameter.

with high error in the standard deviation on the phase, but low error on the fit performed on the power, and vice versa, constitute less than $10 \%$ of the data and are part of the unclassified ACFs.

In order to apply a quantitative test to the different classes, we have set threshold values in the phase and power standard deviations of 0.3 and 0.15 , respectively. Values greater than these thresholds cause the standard deviations to be classified as high. In the same way, one considers that the spectral width is high when it is greater than $200 \mathrm{~m} / \mathrm{s}$. These thresholds have been arbitrarily defined by considering simulation results on the effect of low-frequency wave activity to the ACF (André et al., 2000a) and by looking at ACFs and Doppler spectra extracted from the database. Figure 3 shows the probability density of each parameter computed from the whole database, together with their corresponding thresholds (vertical lines).

The spectral width distribution (Fig. 3a) has a cutoff for values lower than $50 \mathrm{~m} / \mathrm{s}$, due to the rejection of ground echoes. The tail of this distribution is well approximated by a combination of a Gaussian and mono-exponential function. The tail of the two other distributions (Figs. $3 b$ and c) is shaped like a mono-exponential function. Note that these plots describe only the context of the applied thresholds, while any discussion on their shape with respect to a typical distribution function of random processes is beyond the scope of this paper.

\subsection{Results}

Each ACF in the database has been classified according to this scheme. From this, one can deduce for each point of the MLT, MLAT grid, the probability of measuring an ACF of a particular class. The total number of ACFs that could not be classified into one of the three categories was $35 \%$, and these have been removed before calculating the probabilities.

Figure 4a displays the distribution of class " $m$ " (multicomponent spectra). The probability is greater than $45 \%$ on the dayside in a U-shaped region at magnetic latitudes around $80^{\circ}$ and between 04:00 and 20:00 MLT. Except for in the sector 06:00-12:00 MLT, this probability decreases at higher latitudes. In the nightside, the probability reaches $35 \%$, and goes down to $10 \%$ at latitudes lower than $65^{\circ}$, regardless of the MLT.

The probability of having an ACF defined with a single and narrow component ("S" class) (Fig. 4b) is lower than $30 \%$, except at low latitudes $\left(<70^{\circ}\right)$, and it can reach high values $(>45 \%)$ in the sector 12:00-18:00 MLT at latitudes $<75^{\circ}$.

The probability of having an ACF characterized by a single and broad component in the spectrum (" $\mathrm{T}$ " class) is shown in Fig. 4c. This probability is greater than $40 \%$ at low latitudes between 23:00 and 12:00 MLT. This probability is generally greater than $25 \%$, except for a region at low latitudes between 14:00 and 18:00 MLT.

Figure 4 shows that these probabilities are maximum in different regions of the auroral ionosphere. The coherence of these patterns strongly suggests that the three classes of F-region coherent spectra are driven by different processes that have their energy source in different magnetospheric regions. In order to summarize the results shown in Fig. 4, the dominant ACF class has been computed and plotted on a geomagnetic grid. In each point of this grid, the probability of measuring an ACF that falls in a specific class has been already defined before. The dominant class is the one that has a maximum probability that is greater by at least $5 \%$ than the second most dominant ACF class. When this last condition is not satisfied, one considers that both classes are dominant, if their averaged probability is greater than the ACF class, with the least probability, by at least 5\%. Finally, if this last condition is not satisfied, then no dominant class exists.

Figure 5 shows the distribution of the dominant ACF class over the Northern Hemisphere. Class " $\mathrm{S}$ " is coded in gray, " $T$ " in red and " $\mathrm{m}$ " in yellow, respectively. When the dominant class is a combination of two classes, the colour used corresponds to the mix of the colours associated with these classes (for example, orange for a combination of class " $\mathrm{T}$ " and class " $\mathrm{m}$ " or pink for class " $\mathrm{S}$ " and " $\mathrm{T}$ "). Isocontours of the electric potential in the ionosphere that defines the 

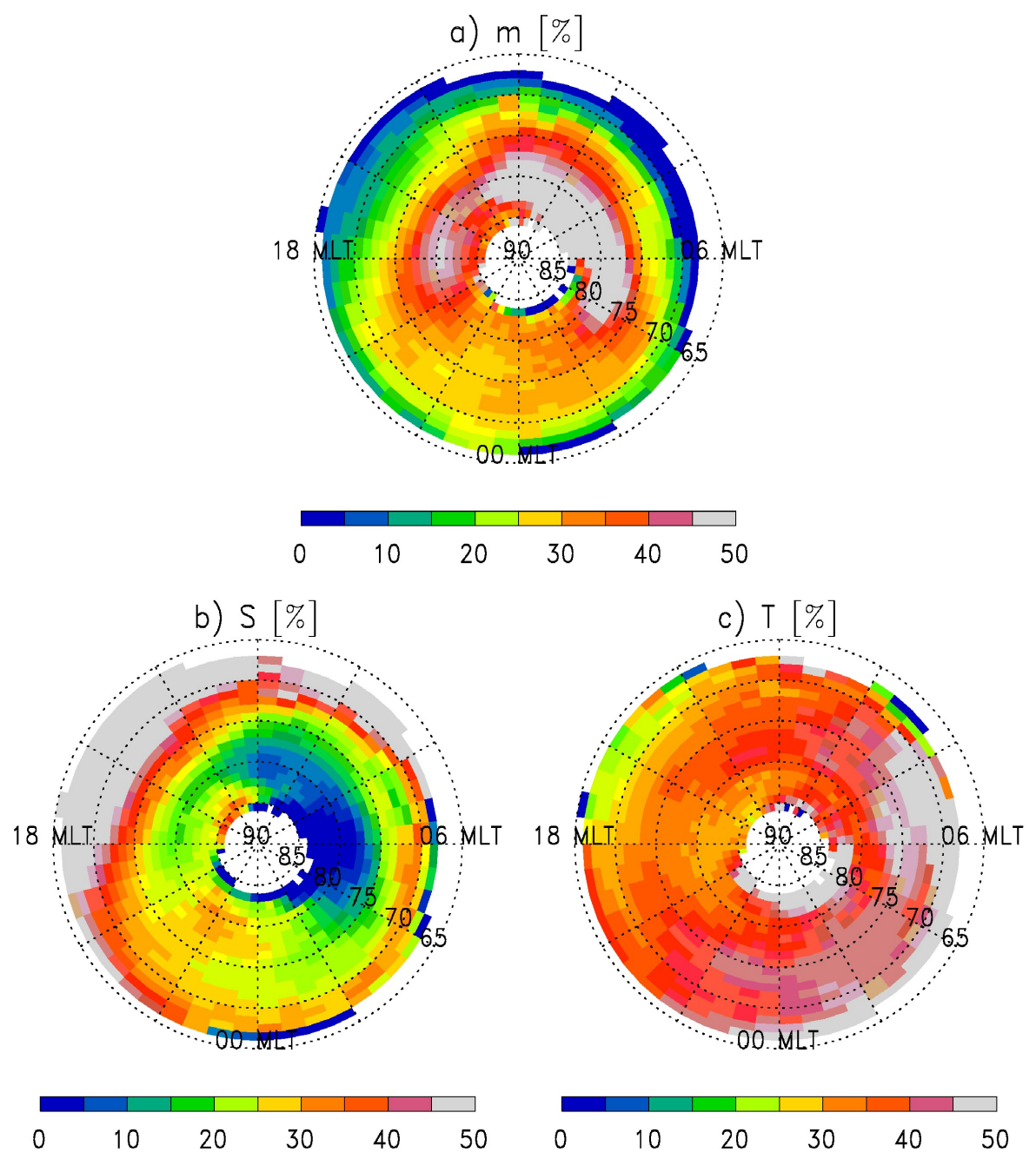

Fig. 4. Occurence of an ACF of (a) class $\mathrm{m},(\mathbf{b})$ class $\mathrm{S}$ and $(\mathbf{c})$ class $(\mathrm{T})$. large-scale convection pattern are superimposed (Heppner and Maynard, 1987, model A). Figure 6 displays isocontours of the average energy of electron precipitation (Hardy et al., 1987) superimposed on the dominant ACF class distribution.

Note that data recorded at very high latitudes have an average spectral width lower than $200 \mathrm{~m} / \mathrm{s}$ (Fig. 2a), whereas the dominant ACF class in this region (Fig. 5) implies a spectral width greater than $200 \mathrm{~m} / \mathrm{s}$. This apparent contradiction is a consequence of the threshold applied to each data distribution, in order to have a better estimate of their mean values. This technique does not affect very much the distribution of ACF class. At high latitudes, the class " $\mathrm{m}$ " is dominant, because the spectral width distribution has a very long tail, which has been removed when computing the mean value. In these regions, ACFs are obtained at a large distance from the radar, and possibly with a $1 \frac{1}{2}$ propagation mode. Several effects, for example, the larger size of the radar cells, might lead to a degradation of the ACF quality, which results in a large spectral width and standard deviation values. We suggest that a part of these spectra are not representative of the typical ACF recorded in this region.

\section{Discussion}

\subsection{Narrow single component spectra (class "S")}

This class (Figs. 4b, 5 and 6 ) is dominant at latitudes lower than $75^{\circ}$ between $14: 00$ and 18:00 MLT and at latitudes lower than $70^{\circ}$ between 10:00 and 20:00 MLT. According to the statistical pattern of the ionospheric convection, this region lies on closed magnetic field lines and is also not associated with a high flux of precipitating particles. The decameter scale irregularities in this region are known to be related to large-scale $(\geq 100 \mathrm{~km}$ ) drifting patches of enhanced ionisation, dubbed auroral blobs (Robinson et al., 1985; Ogawa et al., 1998), which have their origin in polar patches generated in the cusp region and are then convected around to the dusk sector. This scenario is in good agreement with the increase in the " $\mathrm{S}$ " class ACF probability observed in Fig. $4 \mathrm{~b}$ around 22:00 MLT at all magnetic latitudes. Thus, these spectra would be conditioned by the density gradients, which are responsible for the presence of the irregularities, and the resulting turbulent motion of the decameter scale irregularities. 


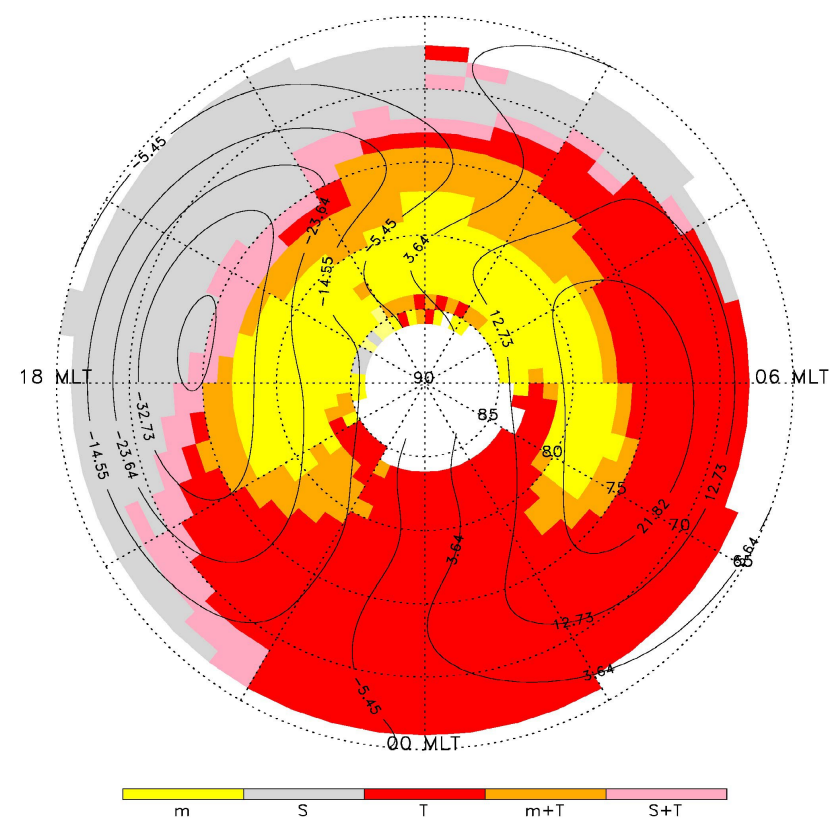

Fig. 5. Convection pattern defined by Heppner and Maynard (1987) (Model A), superimposed on the dominant class of ACF over the auroral zone. Orange and pink correspond to the combination of "T" and "m", and "S" and "T" classes, respectively.

\subsection{Broad single component spectra (class "T")}

Broad single component spectra are dominant in a region collocated with electron precipitation having an average energy of 0.5 to $1.5 \mathrm{keV}$ (Fig. 6). These $1 \mathrm{keV}$ electrons precipitate in the F-region. Thus, they provide an energy source for the growth of irregularities via the current convective instability. Also, they are a source of field-aligned currents that can increase the spectral width if their scale size is lower than the radar range gate. These two effects increase the velocity fluctuations (micro-scale and meso-scale) within one range gate and, therefore, the spectral width (André et al., 2000b).

\subsection{Multi-component spectra (class " $\mathrm{m}$ ")}

This ACF category is dominant in a clear U-shaped region at high latitude predominantly in the dayside ionosphere (approximatively above $77^{\circ}$ magnetic latitude between 06:00 and 18:00 MLT and above $75^{\circ}$ in the bands (03:00-06:00 MLT and 18:00-21:00 MLT). This region includes the cusp where it has been shown that Alfvén wave activity has a crucial impact on the recorded spectra, giving the highest spectral width. Excluding the cusp, the U-shaped region is characterized by a slightly lower spectral width (Fig. 2), but we still consider it likely that low-frequency wave activity is the dominant cause of the multi-component spectra in this region.

Dynamic Explorer 2 has provided useful information on the low-frequency wave activity in the low altitude (300$1000 \mathrm{~km}$ ) auroral zone. Heppner et al. (1993) have investigated the averaged Power Spectral Density (PSD) of elec-

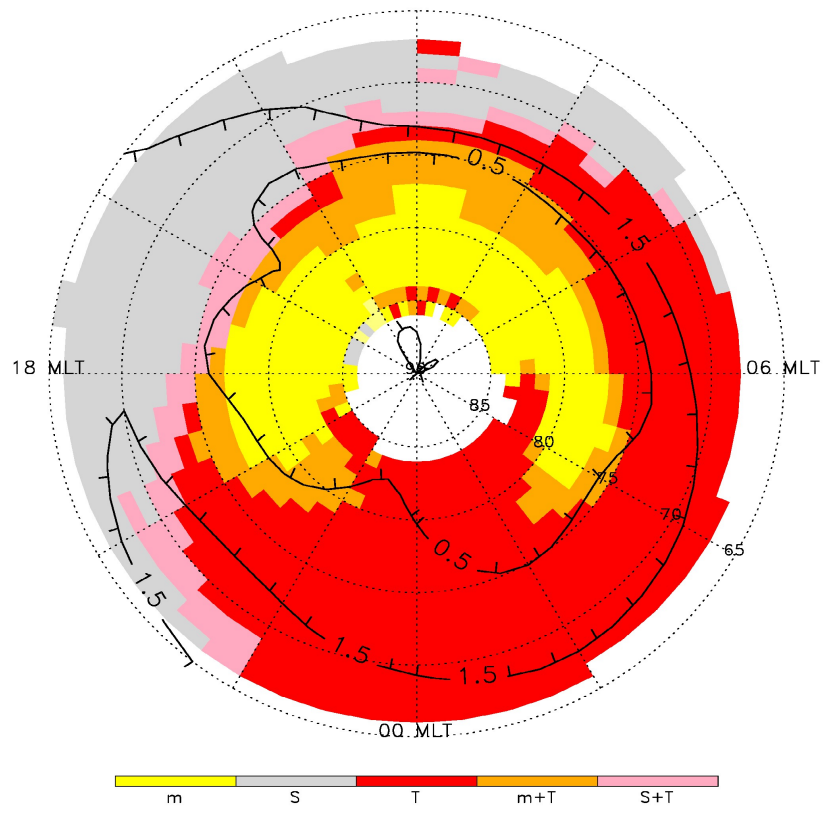

Fig. 6. Isocontours of the average energy of electron precipitation as defined by the model of Hardy et al. (1987), superimposed on the dominant ACF class.

tric field variations perpendicular to the magnetic field in the frequency channel $4-8 \mathrm{~Hz}$, as a function of season, magnetic latitude and local time. The upper left-hand panel of their plate 2 displays a high PSD in a region that could correspond to the auroral oval, with the highest values being located between 05:00 and 16:00 MLT centered around $75^{\circ}$ magnetic latitude (cusp and morning sector). Under the assumption that these low-frequency electric field variations are attributed to an electromagnetic wave (and not to smallscale and static field-aligned currents), one can conclude that such a low-frequency wave activity could be the main source of our multi-component spectra, at least in the morning sector. One has to mention that the observed PSD around $6 \mathrm{~Hz}$ strongly decreases at latitudes greater than $80^{\circ}$, whereas the probability of having an "m" class ACF is still high.

Kivanc and Heelis (1998) have conducted a similar statistical study, concentrating on the PSD of the velocity squared fluctuations around $6 \mathrm{~Hz}$, using the same satellite data. Their results are similar to those obtained by Heppner et al. (1993). They have separated data according to IMF orientations and seasons. In doing so, they have shown a clear shift of the high PSD-region towards higher latitudes when the IMF is directed northward in the winter hemisphere. They have also found a general increase in the recorded power, especially inside the polar cap. Unfortunately, they do not give any average values of the IMF $B_{z}$ for comparison with our study. Our averaged $B_{z}$ (and $B_{y}$ ) is very small and slightly negative $\left(B_{z} \approx-0.3 \mathrm{nT}\right)$. IMF $B_{z}$ values are normally distributed; this means that a significant proportion of ACFs has been recorded under northward or very small $B_{z}$ IMF conditions. Auroral oval precipitations are probably the main source of 
irregularities seen by radar. During $B_{z}$ northward conditions, the contraction of the auroral oval, therefore, enhances the probability of observing scatters at high latitudes. As a consequence, most data observed at high latitudes have statistically been recorded during northward IMF conditions, i.e. when the wave activity is enhanced in the polar cap (Kivanc and Heelis, 1998).

However, it should be noted that the radar spectra do not exhibit the characteristics of a dominant multi-component spectra. The intensity of waves is important. Kivanc and Heelis (1998) have shown a much more intense wave activity in the dayside than in the nightside that should explain the radar spectra behaviour. Another factor is that the nightside corresponds to structured electron precipitation that should also contribute to the electric (or plasma velocity) variations detected by DE-2. Therefore, part of the region (e.g. the dayside) of high PSD at low frequency seen in DE-2 data may have to be interpreted as wave activity, whereas other parts (the nightside) should be interpreted as small-scale fieldaligned currents. This explanation follows the conclusions of Heppner et al. (1993) and could be checked only by multipoint measurements, such as the ones that will be performed by the CLUSTER II mission.

Finally, it is interesting to notice that the equatorward boundary of this $\mathrm{U}$-shaped region seems to be collocated with the ionospheric footprint of the magnetopause. This region is also likely to be a source of the low-frequency Alfvén waves that can propagate down to the ionosphere (e.g. Gurnett et al., 1984; Gurnett, 1991; Lysak, 1999).

\subsection{Typical examples}

Finally, these findings are also observed on individual radar scans and not only through the statistical analysis. Figure 7 presents such a scan, recorded by the Stokkseyri radar on 18 February 1998 at 22:06 UT (19:30 MLT), together with the data sorted by ACF class. In this scan, around $57 \%$ of data have been successfully classified, with each class having about $20 \%$ of the points. The majority of unclassified data (33\%) have a high spectral width, and a large error on the phase estimation, but also an error on the power lower than the threshold used. In these particular ACFs, the power falls rapidly down into the noise (high spectral width). The first points of the ACF contribute to a well-defined phase, but the others add a high fluctuation level, increasing the standard deviation associated with the phase.

Figure 7a displays a general tendency to find the ACF class "S" at low latitudes, the class " $\mathrm{m}$ " at very high latitudes and the class "T" in between. Figures $7 \mathrm{~b}$ and $\mathrm{c}$ show one example of an ACF phase (left) and power (right) falling into the "m" class, measured at 74.2 MLAT and 19:30 MLT. Both phase and power are clearly modulated by at least one other velocity component in the spectrum. A typical ACF of the "T" class appears in Figs. 7d and 7e. Here, the temporal evolution of the phase is linear and the fit on the temporal evolution of the power is better when using an exponential function. Despite this last characteristic, one can easily see that the ACF power starts as a Gaussian function and ends as an exponential one. This has been interpreted in terms of transport characteristics of irregularities (Hanuise et al., 1993; Villain et al., 1996; André et al., 1998). Figures $7 f$ and $g$ show an example of an "S" class ACF that defines a single and narrow component spectrum. These examples come from regions that are approximately located at the same distance from the radar, excluding any propagation effect on the ACF determination. Therefore, this example shows that the ACFs' characteristics are sorted by magnetic latitude rather than by radar range. This observation confirms that ACF characteristics seem to be driven by several individual processes that have their energy source in different regions of the magnetosphere/ionosphere system.

\section{Conclusion}

In this study, we have examined in detail the characteristics of coherent spectra recorded by the SuperDARN radar network. This has been done by building a database compiled from radar data in the Northern Hemisphere over a period of 2 consecutive winters. Each autocorrelation function has been classified according to its characteristics, and located in magnetic latitude and local time. In doing so, one has shown that spectra have a specific behaviour that depends on the geomagnetic region where they are recorded. More specifically, it has been observed that:

- Single and narrow component spectra are recorded mainly on closed field lines in the dusk sector and can be associated with large-scale ionisation enhancements transported into this region by the large-scale convection pattern.

- Single and broad component spectra can be associated with a region of intense and structured low energy $(1 \mathrm{keV})$ electron precipitation in the night and morning sector.

- Multiple component spectra are recorded in a U-shaped region centred around the cusp and located between 03:00 and 21:00 MLT above $75^{\circ}$ magnetic latitude. It has been shown that low-frequency wave activity recorded in the cusp can generate these characteristics in the spectra. This result and the low-frequency wave activity observed by Dynamic Explorer suggest that this wave activity can explain our observations in this region.

These results, obtained during winter conditions, will be further validated by compiling a larger database and sorting the data for different solar wind conditions. It will then be possible to observe if the regions identified in this study are subject to a shift in their location, or are modified, in a manner consistent with the various solar wind conditions. Additional work is also required to fully understand the link between soft electron precipitation and the broad Doppler spectra (class "T") associated with it. These results strongly sug- 

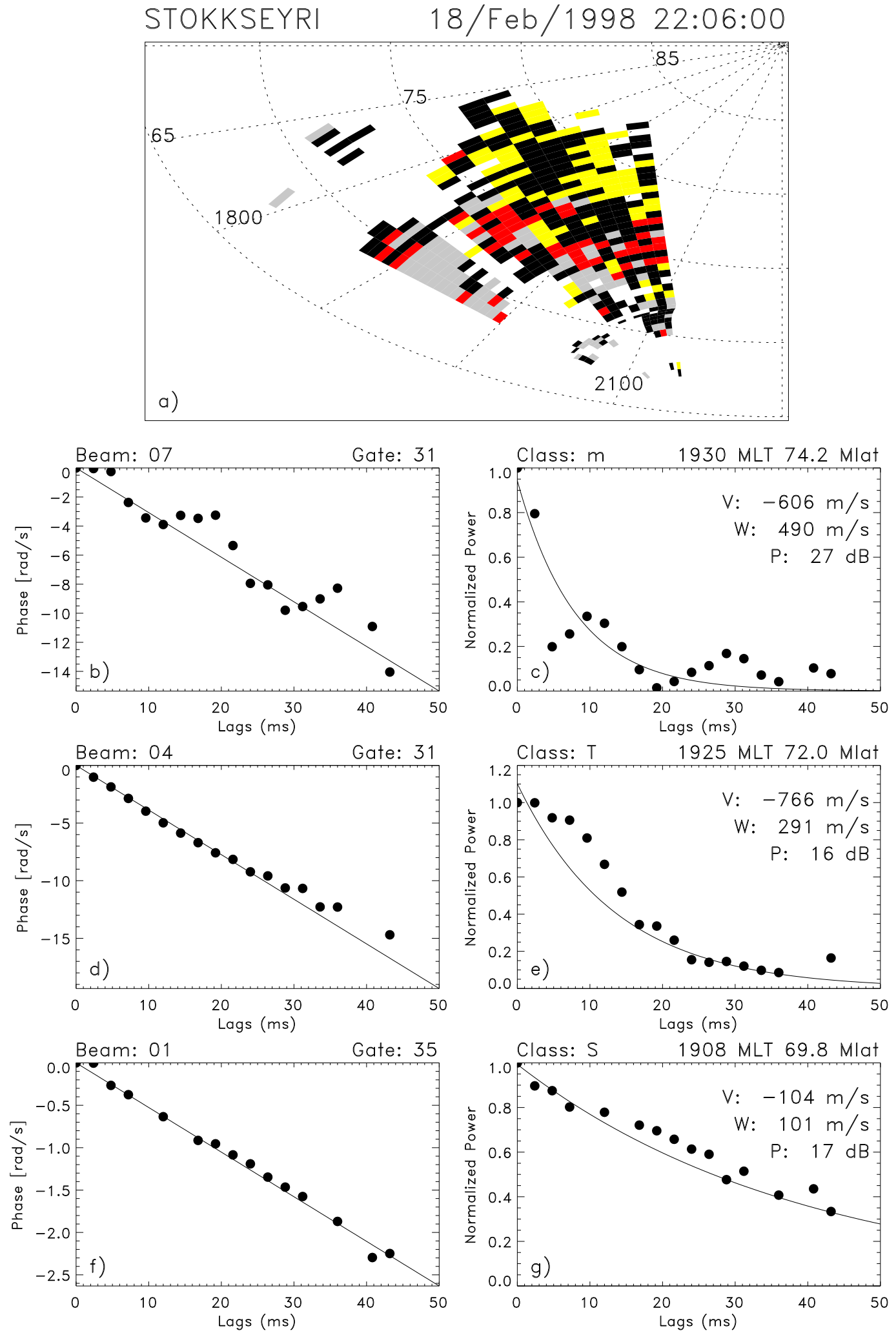

Fig. 7. (a) ACF classes observed by the Stokkseyri radar on the 18 February 1998 at 22:06 UT in geomagnetic (MLT, MLAT) coordinates. Class " $\mathrm{m}$ ", "T" and "S" are coded in yellow, red and gray, respectively. (b) and (c) are the ACF phase and power recorded on beam 7, gate 31 during this scan. In the same way, (d), (e) and (f), (g) are the ACF phase and power recorded on beam 4 gate 31 and beam 1 gate 35 , respectively. 
gest that the SuperDARN radars have the ability to map down magnetospheric regions that drive these energy sources.

Acknowledgements. This work has been funded by the European Community grant ERB4001GT973635. The first author would like to thanks the french Société de Secours des Amis des Sciences for its support. Operation of the Northern Hemisphere SuperDARN radars is supported by the national funding agencies of the U.S., Canada, the U.K., and France. Authors would like to acknowledge NSSDC for providing the auroral oval model and the WIND data.

Topical Editor M. Lester thanks two referees for their help in evaluating this paper

\section{References}

André, R., Grésillon, D., Hanuise, C., and Villain, J. -P.: Auroral ionosphere plasma turbulence transport coefficient: Direct observations from radar coherent backscattering, in: 1998 International Congress on Plasma Physics, (Ed) Pavlo, P., vol. 22 C, pp. 1126-1129, ECA, European Physical Society, 1998.

André, R., Pinnock, M., and Rodger, A. S.: On the SuperDARN autocorrelation function observed in the cusp, Geophys. Res. Lett., 26, 3353-3356, 1999.

André, R., Pinnock, M., and Rodger, A. S.: A physical explanation for the emperically derived signature, J. Geophys. Res., 105, 27 081-27 093, 2000a.

André, R., Pinnock, M., Rodger, A. S., Villain, J.-P., and Hanuise, C.: On the factors conditioning the doppler spectral width determined from SuperDARN HF radars, 2, 77-86, $2000 \mathrm{~b}$.

Baker, K. B. and Wing, S.: A new magnetic coordinate system for conjugate studies at high-latitudes, J.Geophys. Res., 94, 91399147, 1989.

Baker, K. B., Greenwald, R. A., Ruohoniemi, J. M., Dudeney, J. R., Pinnock, M., Newell, P. T., Greenspan, M. E., and Meng, C. I.: Simultaneous HF-radar and DMSP observations of the cusp, Geophys. Res. Lett., 17, 1869-1872, 1990.

Baker, K. B., Dudeney, J. R., Greenwald, R. A., Pinnock, M., Newell, P. T., Rodger, A. S., Mattin, N., and Meng, C. -I.: HF radar signatures of the cusp and low-latitude boundary layer, J. Geophys. Res., 100, 7671-7695, 1995.

Baker, K. B., Rodger, A. S., and Lu, G.: HF-radar observations of the dayside magnetic merging rate: A Geospace Environment Modeling boundary layer campaign study, J. Geophys. Res., 102, 9603-9617, 1997.

Barthes, L., André, R., Cerisier, J. -C., and Villain, J. -P.: Separation of multiple echoes using a high resolution spectral analysis in SuperDARN HF radars, 33, 1005-1017, 1998.

Basu, S.: OGO 6 observations of small-scale irregularity structures associated with subtrough density gradients, J. Geophys. Res., 83, 182-190, 1978.

Dudeney, J. R., Rodger, A. S., Freeman, M. P., Pickett, J., Scudder, J., Sofko, G. J., and Lester, M.: The nightside ionospheric response to IMF $B_{y}$ changes, Geophys. Res. Lett., 25, 26012604, 1998.

Fremouw, E. and Lansinger, J.: Dominant configurations of scintillation-producing irregularities in the auroral zone, J. Geophys. Res., 86, 10 087-10 093, 1981.

Greenwald, R. A., Baker, K. B., Dudeney, J. R., Pinnock, M., Jones, T. B., Thomas, E. C., Villain, J. -P., Cerisier, J. -C., Senior, C., Hanuise, C., Hunsucker, R. D., Sofko, G. J., Koehler, J., Nielsen, E., Pellinen, R., Walker, A. D. M., Sato, N., and Yam- agashi, H.: DARN/SuperDARN: A global view of the dynamics of high-latitude convection, Space Sci. Rev., 71, 761-796, 1995.

Gurnett, D.: Auroral plasma waves, in: Auroral Physics, (Eds) Meng, C.-I., Rycroft, M., and Franck, L., chap. IV-6, pp. 241254, Cambridge Univ. Press, New York, 1991.

Gurnett, D., Huff, R., Menietti, J., Burch, J., Winningham, J., and Shawhan, S.: Correlated low-frequency electric and magnetic noise along the auroral field lines, J. Geophys. Res., 89, 89718985, 1984.

Hanuise, C., Villain, J. -P., Gr'esillon, D., Cabrit, B., Greenwald, R. A., and Baker, K. B.: Interpretation of HF radar Doppler spectra by collective wave scattering theory, Ann. Geophysicae, 11, 29-39, 1993.

Hardy, D., Gussenhoven, M., Raistrick, R., and McNeil, W.: Statistical and functional representations of the pattern of auroral energy flux and conductivity, J. Geophys. Res., 92, 12 275-12 294, 1987.

Heppner, J.: Polar-cap electric field distributions related to the interplanetary magnetic field direction, J. Geophys. Res., 77, 48774887, 1972.

Heppner, J. P. and Maynard, N. C.: Empirical high-latitude electric field models, J. Geophys. Res., 92, 4467-4489, 1987.

Heppner, J., Liebrecht, M., Maynard, N., and Pfaff, R.: Highlatitude distribution of plasma waves and spatial irregularities from DE 2 alternating current electric field observations, J. Geophys. Res., 98, 1629-1652, 1993.

Holzworth, R. and Meng, C. -I.: Mathematical representation of the auroral oval, Geophys. Res. Lett., 2, 377-380, 1975.

Huber, M. and Sofko, G.: Small-scale vortices in the high-latitude F- region, J. Geophys. Res., 105, 20 885-20 898, 2000.

Kivanç, Ö. and Heelis, R.: Spatial distribution of ionospheric plasma and field structures in the high-latitude F-region, J. Geophys. Res., 103, 6955-6968, 1998.

Lester, M., Milan, S., Besser, V., and Smith, R.: A case study of HF radar spectra and $630.0 \mathrm{~nm}$ auroral emission in the pre-midnight sector, Ann. Geophys., 19, 327-339, 2001.

Lysak, R.: Propagation of Alfvén waves throughout the ionosphere: Dependence on ionospheric parameters, J. Geophys. Res., 104, 10017-10030, 1999.

Milan, S. E., Lester, M., Cowley, S. W. H., Moen, J., Sandholt, P. E., and Owen, C. J.: Meridian-scanning photometer, coherent HF radar, and magnetometer observations of the cusp: a case study, Ann. Geophysicae, 17, 159-172, 1999.

Ogawa, T., Nishitani, N., Pinnock, M., Sato, N., Yamagishi, H., and Yukimatu, A.: Antarctic HF radar observations of irregularities associated with polar patches and auroral blobs: a case study, J. Geophys. Res., 103, 26 547-26 558,1998.

Opgenoorth, H. J., Pellinen, R. J., Baumjohann, W., Nielsen, E., Marklund, G., and Eliasson L.: Three-dimensional current flow and particle precipitation in a westward travelling surge (observed during the BARIUM-GEOS rocket experiment), J. Geophys. Res., 88, 3138-3152, 1983.

Pinnock, M., Rodger, A. S., Baker, K. B., Lu, G., and Hairston, M.: Conjugate observations of the day-side reconnection electric field: A GEM boundary layer campaign, Ann. Geophysicae, 17, 443-454, 1999.

Robinson, R., Tsunoda, R., Vickrey, J., and Guerin, L.: Sources of F-region ionization enhancements in the nighttime auroral zone, J. Geophys. Res., 90, 7533-7546, 1985.

Rodger, A. S., Mende, S. B., Rosenberg, T. J., and Baker, K. B.: Simultaneous optical and HF radar observations of the ionospheric cusp, Geophys. Res. Lett., 22, 2045-2048, 1995. 
Ruohoniemi, J. M. and Greenwald, R. A.: Rates of scattering occurence in routine HF radar observations during solar cycle maximum, J. Geophys. Res., 32, 1051-1070, 1997.

Ruohoniemi, J., Greenwald, R., Villain, J. -P., Baker, K., Newell, P., and Meng, C. -I.: Coherent HF radar backscatter from smallscale irregularities in the dusk sector of subauroral ionosphere, J. Geophys. Res., 93, 12 871-12 882, 1988.

Schiffler, A., Sofko, G., Newell, P., and Greenwald, R.: Mapping the outer LLBL with SuperDARN double peaked spectra, Geophys. Res. Lett., 24, 3149-3152, 1997.

Villain, J.-P., Caudal, G., and Hanuise, C.: A SAFARI-EISCAT comparison between the velocity of F-region small-scale irregularities and the ion drift, J. Geophys. Res., 90, 8433-8443, 1985.

Villain, J.-P., Greenwald, R. A., Baker, K. B., and Ruohoniemi, J. M.: HF radar observations of E-region plasma irregularities produced by oblique electron streaming, J. Geophys. Res., 92, 12327-12342, 1987.

Villain, J.-P., Andrè, R., Hanuise, C., and Grésillon, D.: Observation of the high-latitude ionosphere by HF radars: Interpretation in terms of collective wave scattering and characterization of turbulence, J. Atmos. Terr. Phys., 58, 943-958, 1996. 